\title{
Graphs Drawn with Few Crossings per Edge
}

\author{
János Pach ${ }^{\star}$ and Géza Tóth ${ }^{\star \star}$ \\ Courant Institute, New York University \\ 251 Mercer Street, New York, NY 10012
}

\begin{abstract}
We show that if a graph of $v$ vertices can be drawn in the plane so that every edge crosses at most $k>0$ others, then its number of edges cannot exceed $4.108 \sqrt{k} v$. For $k \leq 4$, we establish a better bound, $(k+3)(v-2)$, which is tight for $k=1$ and 2 . We apply these estimates to improve a result of Ajtai et al. and Leighton, providing a general lower bound for the crossing number of a graph in terms of its number of vertices and edges.
\end{abstract}

\section{Introduction}

Given a simple graph $G$, let $v(G)$ and $e(G)$ denote its number of vertices and edges, respectively. We say that $G$ is drawn in the plane if its vertices are represented by distinct points of the plane and its edges are represented by Jordan arcs connecting the corresponding point pairs but not passing through any other vertex. Throughout this paper, we only consider drawings with the property that any two arcs have at most one point in common. This is either a common endpoint or a common interior point where the two arcs properly cross each other. We will not make any notational distinction between vertices of $G$ and the corresponding points in the plane, or between edges of $G$ and the corresponding Jordan arcs.

We address the following question. What is the maximum number of edges that a simple graph of $v$ vertices can have if it can be drawn in the plane so that every edge crosses at most $k$ others? For $k=0$, i.e. for planar graphs, the answer is $3 v-6$. Our first theorem generalizes this result to $k \leq 4$. The case $k=1$ has been discovered independently by Bernd Gärtner, Torsten Thiele, and Günter Ziegler (personal communication).

Theorem 1. Let $G$ be a simple graph drawn in the plane so that every edge is crossed by at most $k$ others. If $0 \leq k \leq 4$, then we have

$$
e(G) \leq(k+3)(v(G)-2) \text {. }
$$

For $k=0,1,2$, the above bound cannot be improved (see Remark 2.3 at the end of the next section.)

The crossing number $\operatorname{cr}(G)$ of a graph $G$ is the minimum number of crossing pairs of edges, over all drawings of $G$ in the plane.

\footnotetext{
* Supported by NSF grant CCR-94-24398 and PSC-CUNY Research Award 667339

* Supported by OTKA-T-020914 and OTKA-14220.
} 
Ajtai et al. [AC82] and, independently, Leighton [L83] obtained a general lower bound for the crossing number of a graph, which found many applications in combinatorial geometry and in VLSI design (see [PA95], [PS96], [S95]). Our next result, whose proof is based on Theorem 1, improves the bound of Ajtai et al. by roughly a factor of 2 .

Theorem 2. The crossing number of any simple graph $G$ satisfies

$$
\operatorname{cr}(G) \geq \frac{1}{33.75} \frac{e^{3}(G)}{v^{2}(G)}-0.9 v(G)>0.029 \frac{e^{3}(G)}{v^{2}(G)}-0.9 v(G) .
$$

Theorem 3. Let $G$ be a simple graph drawn in the plane so that every edge is crossed by at most $k$ others, for some $k \geq 1$. Then we have

$$
e(G) \leq \sqrt{16.875 k} v(G) \approx 4.108 \sqrt{k} v(G) .
$$

Theorems 2 and 3 do not remain true if we replace the constants 0.029 and 4.108 by 0.06 and 1.92 , respectively (see Remarks 3.2 and 3.3 ).

In the last section, we use the ideas of Székely [S95] to deduce some consequences of Theorem 2.

\section{Proof of Theorem 1}

First we need a lemma for multigraphs, i.e., for graphs that may have multiple edges. In a drawing of a multigraph, any two non-disjoint edges either share only endpoints or have precisely one point in common, at which they properly cross.

Let $M$ be a multigraph drawn in the plane so that every edge crosses at most $k$ other edges. Let $M^{\prime}$ be a sub-multigraph of $M$ with the largest number of edges such that in the drawing of $M^{\prime}$ (inherited from the drawing of $M$ ), no two edges cross each other. We say that $M^{\prime}$ is a maximal plane sub-multigraph of $M$, and its faces will be denoted by $\Phi_{1}, \Phi_{2}, \ldots, \Phi_{m}$. Let $\left|\Phi_{i}\right|$ denote the number of edges of $M^{\prime}$ along the boundary of $\Phi_{i}$, where every edge whose both sides belong to the interior of $\Phi_{i}$ is counted twice. It follows from the maximality of $M^{\prime}$ that every edge $e$ of $M-M^{\prime}$ crosses at least one edge of $M^{\prime}$. The closed portion between an endpoint of $e$ and the nearest crossing of $e$ with an edge of $M^{\prime}$ is called a half-edge. Thus, every edge of $M-M^{\prime}$ contains two half-edges. Every half-edge lies in a face $\Phi$ and intersects at most $k-1$ other half-edges and an edge of $\Phi_{i}$ (not counting the incidences at the vertices of $M$ ). Let $h\left(\Phi_{i}\right)$ denote the number of half-edges in $\Phi_{i}$.

Lemma 2.1. Let $0 \leq k \leq 4$ and let $M$ be a multigraph drawn in the plane so that every edge crosses at most $k$ others. Let $M^{\prime}$ be a maximal plane sub-multigraph of $M$, and let $\Phi$ denote a face with $|\Phi|=s \geq 3$ sides in $M^{\prime}$, whose bondary is connected.

Then the number of half-edges in $\Phi$ satisfies

$$
h(\Phi) \leq(s-2)(k+1)-1 .
$$


Proof: We proceed by induction on $s$. First, let $s=3$ and denote the vertices of $\Phi$ by $A, B$, and $C$. Let $a, b$, and $c$ denote the number of half-edges in $\Phi$ emanating from $A, B$, and $C$, respectively. We have to show that $a+b+c \leq k$. For $k=0$, there is nothing to prove. We check the cases $k=1,2,3,4$, separately.

- $k=1$ : If $a=b=c=0$, we are done. Assume without loss of generality that $a \geq 1$. But then $a=1$, because all half-edges in $\Phi$ emanating from $A$ intersect the edge $B C$. Since any half-edge in $\Phi$ emanating from $B$ or $C$ would create another intersection on the half-edge starting from $A$, we obtain $b=c=0$. Hence, $a+b+c=1$.

- $\boldsymbol{k}=2$ : Suppose without loss of generality that $a \geq 1$. Clearly, $a \leq 2$. If $a=1$, the unique half-edge in $\Phi$ emanating from $A$ intersects all half-edges coming from $B$ and $C$. So $1+b+c=a+b+c \leq 2$. If $a=2$, any half-edge from $B$ would intersect both half-edges emanating from $A$ and the edge $A C$, which is impossible. Hence, $b=0$. Similarly, $c=0$, and $a+b+c=2$.

- $k=3$ : Just like before, we can exclude all cases when $a+b+c>3$, except for the case $a=b=2$ and $c=0$. Now let $e_{1}$ and $e_{2}$ denote the edges containing the two half-edges in $\Phi$ emanating from $A$. Both of them intersect the two halfedges starting from $B$ and the edge $B C$. So they cannot cross any other edge. Removing $B C$ from $M^{\prime}$ and adding $e_{1}$ and $e_{2}$, we would obtain a larger plane sub-multigraph of $M$, contradicting the maximality of $M^{\prime}$.

- $k=4$ : We can again exclude all cases when $a+b+c>4$, with the exception of the case $a=2, b=3, c=0$. As before, let $e_{1}$ and $e_{2}$ denote the edges containing the two half-edges in $\Phi$ emanating from $A$. Now both $e_{1}$ and $e_{2}$ are intersected by the three half-edges emanating from $B$ and by the edge $B C$. Hence, there are no other edges crossing them, and the number of edges of $M^{\prime}$ can be increased by replacing $B C$ with $e_{1}$ and $e_{2}$. Contradiction.

Now let $s>3$, and suppose that the lemma has already been proved for faces with fewer than $s$ sides. Let $A_{1}, A_{2}, \ldots, A_{s}$ denote the sequence of vertices of $\Phi$, listed in clockwise order. In this sequence, the same vertex may occur several times (as many times as it is visited during a full clockwise tour around the boundary of $\Phi)$. For simplicity, let $A_{0}=A_{s}$ and $A_{s+1}=A_{1}$.

We call an open arc empty if it does not intersect any half-edge in $\Phi$.

Case 1. Assume that there is a half-edge $e=A_{i} E$ in $\Phi$, where $E$ is an interior point of the side $A_{j} A_{j+1}$, and either

(i) the $\operatorname{arc} A_{j} E \subseteq A_{j} A_{j+1}$ is empty and $i \neq j-1$, or

(ii) the arc $E A_{j+1} \subseteq A_{j} A_{j+1}$ is empty and $i \neq j+2$.

By symmetry, we can suppose that $e$ satisfies (i). Let $M^{*}$ denote the (multigraph) drawing obtained from $M$ by replacing the edge of $M$ containing $e$ with a new edge $e^{\prime}=A_{i} A_{j}$ lying in $\Phi$ and running very close to $e$ and the arc $A_{j} E$.

Since $A_{j} E$ was empty in $M, e^{\prime}$ crosses exactly the same half-edges as $e$. Thus, $M^{*}$ also satisfies the condition that each of its edges crosses at most $k$ other edges. Clearly, $M^{\prime} \cup e^{\prime}$ is a maximal plane sub-multigraph of $M^{*}$. In $M^{*}$, $e^{\prime}$ divides $\Phi$ into two faces $\Phi^{\prime}$ and $\Phi^{\prime \prime}$ having $s^{\prime}$ and $s^{\prime \prime}$ sides, respectively, where $3 \leq s^{\prime}, s^{\prime \prime}<s, s^{\prime}+s^{\prime \prime}=s+2$. 
Each half-edge in $\Phi$, except $e$, corresponds to a half-edge in $\Phi^{\prime}$ or $\Phi^{\prime \prime}$. By the induction hypothesis,

$h(\Phi)=h\left(\Phi^{\prime}\right)+h\left(\Phi^{\prime \prime}\right)+1 \leq\left(s^{\prime}-2\right)(k+1)+\left(s^{\prime \prime}-2\right)(k+1)-2+1=(s-2)(k+1)-1$.
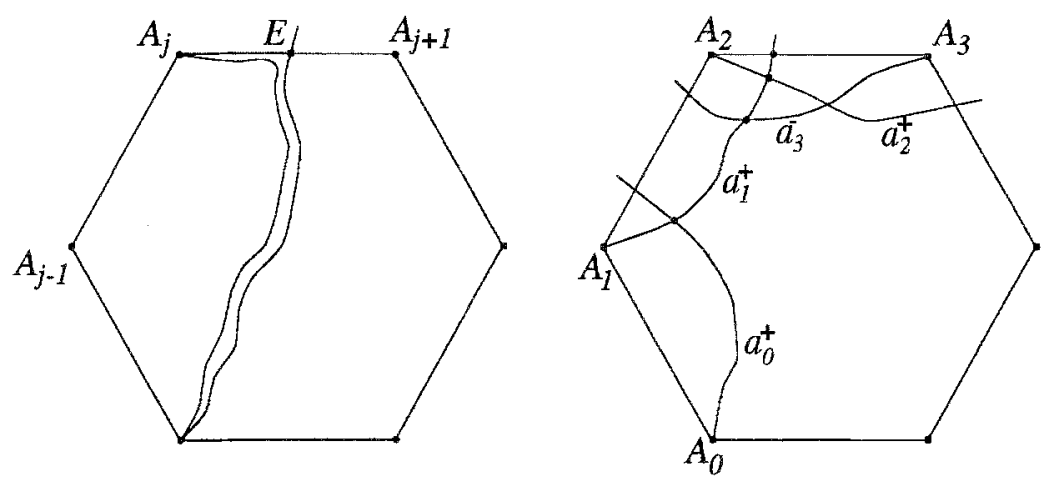

Figure 1.

Case 2. Assume that there is no half-edge in $\Phi$ that satisfies the condition of Case 1.

Then, for any non-empty side $A_{i} A_{i+1}$ of $\Phi$, the half-edge $a_{i-1}^{+}$(resp. $a_{i+2}^{-}$) whose intersection with $A_{i} A_{i+1}$ is closest to $A_{i}$ (resp. closest to $A_{i+1}$ ) starts at the vertex $A_{i-1}$ (resp. $A_{i+2}$ ).

Since any side of $\Phi$ intersects at most $k$ half-edges, if there are two empty sides of $\Phi$, then $h(\Phi) \leq(s-2) k \leq(s-2)(k+1)-1$. So we can suppose that $\Phi$ has at most one empty side. Since $s>3$, there are three consecutive non-empty sides, say, $A_{1} A_{2}, A_{2} A_{3}$, and $A_{3} A_{4}$.

Then $a_{1}^{+}$must intersect $a_{0}^{+}, a_{2}^{+}, a_{3}^{-}$, and the side $A_{2} A_{3}$. Similarly, $a_{4}^{-}$must intersect $a_{5}^{-}, a_{3}^{+}, a_{2}^{+}$, and the side $A_{2} A_{3}$. This is clearly impossible if $k=1,2$ or 3.

For $k=4$, let $e_{1}$ and $e_{2}$ denote the edges of $M$ containing $a_{1}^{+}$and $a_{4}^{-}$, respectively. Both of these edges cross three half-edges and the side $A_{2} A_{3}$ of $\Phi$, so neither of them can cross any further edges. Removing the edge $A_{2} A_{3}$ from $M^{\prime}$ and adding $e_{1}$ and $e_{2}$, we would obtain a plane sub-multigraph of $M$, whose number of edges is larger than the number of edges of $M^{\prime}$. This contradicts the maximality of $M^{\prime}$, completing the proof of Lemma 2.1.

For any face $\Phi$ with at least 3 sides, let $t(\Phi)$ denote the number of triangles in a triangulation of $\Phi$.

Lemma 2.2 Let $\Phi$ be any face of $M^{\prime}$ with $|\Phi| \geq 3$ sides. Then the number of half-edges of $\Phi$ satisfies

$$
h(\Phi) \leq t(\Phi) k+|\Phi|-3 .
$$

Proof: If the boundary of $\Phi$ is connected, then $t(\Phi)=|\Phi|-2$. Hence, by Lemma $2.1, h(\Phi) \leq(|\Phi|-2)(k+1)-1=t(\Phi) k+|\Phi|-3$. 
For any face $\Phi$, the number of half-edges in $\Phi$ is at most $|\Phi| k$, because every side of $\Phi$ intersects at most $k$ half-edges. If the boundary of $\Phi$ is not connected, then $t(\Phi) \geq|\Phi|$. Therefore, in this case, we have $h(\Phi) \leq|\Phi| k \leq t(\Phi) k+|\Phi|-3$.

Now we are ready to prove Theorem 1. Suppose that a simple graph $G$ is drawn in the plane with at most $k$ crossings on each edge. Let $G^{\prime}$ be a maximal plane subgraph of $G$. Denote the faces of $G^{\prime}$ by $\Phi_{1}, \Phi_{2}, \ldots \Phi_{m}$. To triangulate $\Phi_{i}$, we need at least $\left|\Phi_{i}\right|-3$ edges. Therefore,

$$
e\left(G^{\prime}\right) \leq 3 v-6-\sum_{i=1}^{m}\left(\left|\Phi_{i}\right|-3\right) .
$$

Every edge of $G-G^{\prime}$ gives rise to two half-edges. So, Lemma 2.2 yields that

$$
e\left(G-G^{\prime}\right) \leq \frac{1}{2} \sum_{i=1}^{m}\left(t\left(\Phi_{i}\right) k+\left|\Phi_{i}\right|-3\right) .
$$

Summing up the last two inequalities and noticing that the total number of triangles satisfies $\sum_{i} t\left(\Phi_{i}\right)=2 v(G)-4$, we obtain

$$
\begin{gathered}
e(G) \leq 3 v(G)-6+\frac{1}{2} \sum_{i=1}^{m}\left(t\left(\Phi_{i}\right) k-\left(\left|\Phi_{i}\right|-3\right)\right) \leq 3 v(G)-6+(v(G)-2) k \\
=(k+3)(v(G)-2)
\end{gathered}
$$

as desired. $\square \square$

Remark 2.3. For $k=0$, the bound $e \leq 3 v-6$ is tight for any triangulation.

For $k=1$, the obtained bound $e \leq 4 v-8$ is also tight, provided that $v \geq 12$. First we show that for every $v \geq 12$ there is a planar graph with $v$ vertices, all of whose faces are quadrilaterals, and no two faces share more than one edge. Indeed, Figure 2 illustrates that such graphs exist for $v=8,13,14,15$. Once we have an example $G$ with $v$ vertices, we can construct another one with $v+4$ vertices, by replacing some face of $G$ by the 8-point example in Figure 2. Notice that if we add both diagonals of each face (including the external face), then we obtain a graph with $4 v-8$ edges such that along each edge there is at most one crossing.
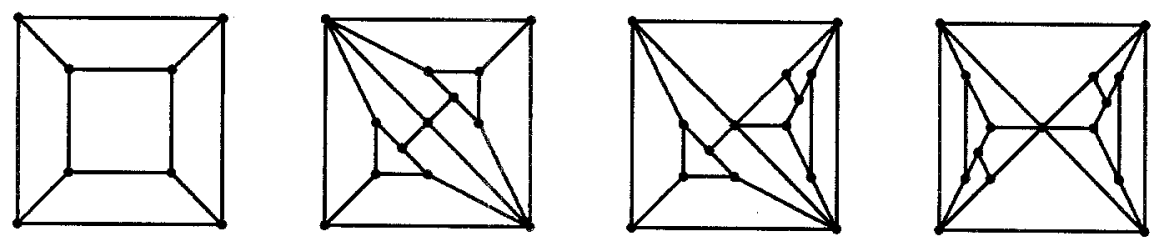

Figure 2. 
For $k=2$, the bound $e \leq 5 v-10$ is sharp for all $v \geq 50$ such that $v \equiv$ $2(\bmod 3)$. For simplicity, we only exhibit a construction for $v \equiv 5(\bmod 15)$. First we construct a planar graph whose faces are pentagons and two faces have at most one edge in common. For $v=20$, such a graph is shown in Figure 3 . The number of vertices of such an example $G$ can be increased by 15 , by replacing some face of $G$ with the graph depicted in Figure 3. Notice that if we add all 5 diagonals of each face to $G$, then we obtain a graph with $v$ vertices and $5 v-10$ edges, in which every edge crosses at most 2 others.

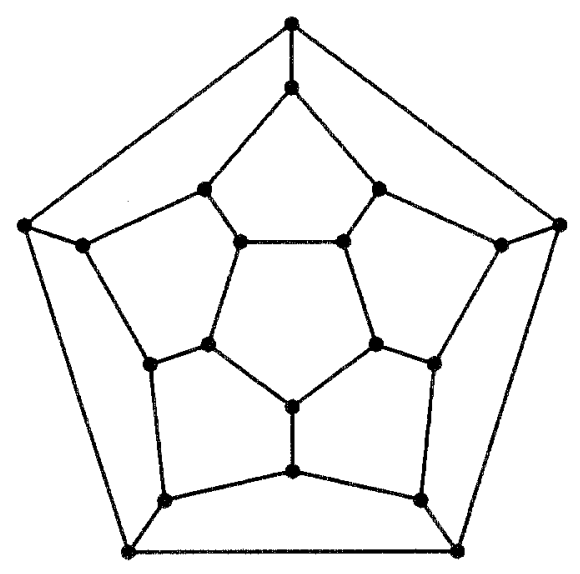

Figure 3.

\section{Proofs of Theorems 2 and 3}

In this section, we slightly improve the best known general lower bound on the crossing number of a graph, due to Ajtai et al. and Leighton. Our proof is based on the following consequence of Theorem 1.

Corollary 3.1. The crossing number of any simple graph $G$ with at least 3 vertices satisfies

$$
\operatorname{cr}(G) \geq 5 e(G)-25 v(G)+50 .
$$

Proof: If $e(G) \leq 3 v(G)-6$, then the statement is void. Assume $e(G)>3 v(G)-6$.

It follows from Theorem 1 that if $e(G)>(k+3)(v(G)-2)$, then $G$ has an edge crossed by at least $k+1$ other edges $(k \leq 4)$. Deleting such an edge, we obtain by induction on $e(G)$ that the number of crossings is at least

$$
\sum_{k=0}^{4}[e(G)-(k+3)(v(G)-2)]=5 e(G)-25 v(G)+50 .
$$

Proof of Theorem 2. Let $G$ be a simple graph drawn in the plane with $\operatorname{cr}(G)$ crossings, and suppose that $e(G) \geq 7.5 v(G)$. 
Construct a random subgraph $G^{\prime} \subseteq G$ by selecting each vertex of $G$ independently with probability $p=7.5 v(G) / e(G) \leq 1$, and letting $G^{\prime}$ be the subgraph induced by the selected vertices. The expected number of vertices of $G^{\prime}$, $E\left[v\left(G^{\prime}\right)\right]=p v(G)$. Similarly, $E\left[e\left(G^{\prime}\right)\right]=p^{2} e(G)$. The expected number of crossings in the drawing of $G^{\prime}$ inherited from $G$ is $p^{4} \operatorname{cr}(G)$, and the expected value of the crossing number of $G^{\prime}$ is even smaller.

By Corollary 3.1, $\operatorname{cr}\left(G^{\prime}\right) \geq 5 e\left(G^{\prime}\right)-25 v\left(G^{\prime}\right)$ for every $G^{\prime}$. Taking expectations,

$$
p^{4} \operatorname{cr}(G) \geq E\left[\operatorname{cr}\left(G^{\prime}\right)\right] \geq 5 E\left[e\left(G^{\prime}\right)\right]-25 E\left[v\left(G^{\prime}\right)\right]=5 p^{2} e(G)-25 p v(G) .
$$

This implies that

$$
\operatorname{cr}(G) \geq \frac{1}{33.75} \frac{e^{3}(G)}{v^{2}(G)}
$$

whenever $e(G) \geq 7.5 v(G)$. With a slight modification, we can extend this bound to the range $e(G)<7.5 v(G)$. In fact, using Corollary 3.1 , it is easy to check that

$$
\operatorname{cr}(G) \geq \frac{1}{33.75} \frac{e^{3}(G)}{v^{2}(G)}-0.9 v(G)
$$

is valid for every simple graph $G$.

Proof of Theorem 3. For $k \leq 4$, the result is weaker than the bounds given in Theorem 1.

So let $k>4$, and consider a drawing of $G$ such that every edge crosses at most $k$ others. Let $C$ denote the number of crossings in this drawing. If $e(G)<7.5 v(G)$, then there is nothing to prove. If $e(G) \geq 7.5 v(G)$, then using the stronger form (1) of Theorem 2, we obtain

$$
\frac{1}{33.75} \frac{e^{3}(G)}{v^{2}(G)} \leq \operatorname{cr}(G) \leq C \leq \frac{e(G) k}{2} .
$$

Consequently,

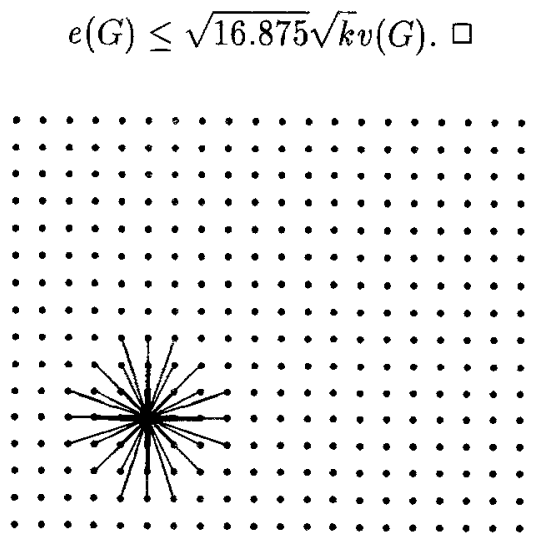

Figure 4. 
Remark 3.2. The bound given in Theorem 2 is asymptotically tight, apart from the values of the constants. The best construction we found is the following.

Let $v \ll e \ll v^{2}$. Let $V(G)$ be a set of $v$ points arranged in a slightly perturbed unit square grid of size $\sqrt{v} \times \sqrt{v}$, so that the points are in general position. Let $d=\sqrt{2 e / \pi v}$, so that $d^{2} \pi=e / 2 v$.

Connect two points by a straight-line segment if and only if their distance is at most $d$. Then $v(G)=v, e(G) \approx v d^{2} \pi / 2=e$.

To count the number of crossings in $G$, let $S(a)=\{(x, y) \mid 1 \leq x, y \leq a\}$, and for any two segments $\left(u_{1}, u_{2}\right),\left(v_{1}, v_{2}\right),\left(u_{1}, u_{2}\right) \otimes\left(v_{1}, v_{2}\right)$ means that the two segments cross each other. Then the number of crossings in $G$ is

$$
\begin{gathered}
\frac{1}{8}\left|\left\{\left(u_{1}, u_{2}, v_{1}, v_{2}\right) \in[V(G)]^{4} \mid\left\|u_{1}-u_{2}\right\|,\left\|v_{1}-v_{2}\right\| \leq d,\left(u_{1}, u_{2}\right) \otimes\left(v_{1}, v_{2}\right)\right\}\right| \\
\approx \frac{1}{8} \int_{u_{1} \in S(\sqrt{v})} \int_{\substack{u_{2} \in S(\sqrt{v}) \\
\left\|u_{1}-u_{2}\right\| \leq d}} \int_{v_{1} \in S(\sqrt{v})} \int_{\substack{v_{2} \in S(\sqrt{v}) \\
\left(u_{1}, u_{2}\right) \otimes\left(v_{1}, v_{2}\right)}} 1 d v_{2} d v_{1} d u_{2} d u_{1} \\
=\frac{2 \pi}{27} v d^{6}(1+o(1)) .
\end{gathered}
$$

Thus,

$$
\operatorname{cr}(G) \leq \frac{2 \pi}{27} v d^{6}(1+o(1)) \approx \frac{16}{27 \pi} \frac{e^{3}}{v^{2}} \approx .06 \frac{e^{3}}{v^{2}} .
$$

J. Spencer [S96] showed that the limit

$$
c=\lim \frac{v^{2}}{e^{3}} \min _{\substack{|V(G)|=v \\|E(G)|=e}} \operatorname{cr}(G)
$$

exists, as $v \rightarrow \infty$ and $v \ll e \ll v^{2}$. By our results, $.06 \geq e \geq .029$.

Remark 3.3. To see that the bound obtained in Theorem 3 is also asymptotically tight. Consider the same construction as in Remark 3.2 , but now set $d=\sqrt[4]{3 k / 2}(1-o(1))$, as $k$ tends to infinity. Just like above, it can be shown that no edge crosses more than $k$ other edges. The number of edges

$$
e(G)=v(G) \frac{d^{2} \pi}{2}(1-o(1))=v(G) \sqrt{k} \frac{\sqrt{3} \pi}{\sqrt{8}}
$$

Thus, we have

$$
1.92(1-o(1)) \sqrt{k} v(G)<\max e(G)<4.108 \sqrt{k} v(G)
$$

where the maximum is taken over all simple graphs with $v(G)$ vertices that have a drawing with at most $k$ crossings per edge. 


\section{Two further applications}

Using Székely's method (see [S95]) and Theorem 2, we can improve the constant in the Szemerédi-Trotter theorem [ST83], [CE90].

Theorem 4.1. Given $m$ points and $n$ lines in the Euclidean plane, the number of incidences between them is at most $2.57 n^{2 / 3} m^{2 / 3}+2.07(m+n)$.

Proof. Define a graph $G$ drawn in the plane such that the vertex set of $G$ is the given set of $m$ points, and join two points with an edge drawn as a straight line segment if the two points are consecutive along one of the lines. Let $I$ denote the total number of incidences between the given $m$ points and $n$ lines. Then $v(G)=m$ and $e(G)=I-n$. Since every edge belongs to one of the $n$ lines, $\operatorname{cr}(G) \leq\left(\begin{array}{c}n \\ 2\end{array}\right)$. Applying Theorem 2 to $G$, we obtain that

$$
\left(\begin{array}{l}
n \\
2
\end{array}\right) \geq \operatorname{cr}(G) \geq \frac{1}{33.75} \frac{(I-n)^{3}}{m^{2}}-0.9 m,
$$

which implies, by Jensen's inequality, that

$$
I \leq 2.57 n^{2 / 3} m^{2 / 3}+3.13 m+n .
$$

By symmetry we also have that

$$
I \leq 2.57 n^{2 / 3} m^{2 / 3}+m+3.13 n .
$$

Taking the average of the last two inequalities, the result follows.

Remark 4.2. As Erdös pointed out fifty years ago, the order of magnitude of the bound in Theorem 4.1 cannot be improved. To see this, one can take $n$ points arranged in a unit square grid of size $\sqrt{n} \times \sqrt{n}$ and consider the $m$ most "popular" lines.

More precisely, for any $1>\varepsilon>0$, take all lines which contain at least $\varepsilon \sqrt{n}$ of the points. Then, for the number of lines $m$ we have

$$
m \approx 4 \sqrt{n} \sum_{r=1}^{1 / \varepsilon} \sum_{\substack{s<r \\(r, s)=1}} r+s-2 r s \varepsilon=6 \sqrt{n} \sum_{r=1}^{1 / \varepsilon} r \phi(r)-4 \sqrt{n} \varepsilon \sum_{r=1}^{1 / \varepsilon} r^{2} \phi(r) \approx \frac{6 \sqrt{n}}{\pi^{2} \varepsilon^{3}} .
$$

Here $\phi(n)$ denotes Euler's function and we used the formula $\sum_{r=1}^{N} \phi(r) \approx 3 N^{2} / \pi^{2}$ (see e. g. [HW54]). By similar calculations, for the number of incidences $I$ we get

$$
I \approx 4 n \sum_{\substack{r=1 \\(r, s)=1}}^{1 / \varepsilon} \sum_{\substack{s<r \\(r)=1}} 1-r s \varepsilon^{2}=4 n \sum_{r=1}^{1 / \varepsilon} \phi(r)-2 n \varepsilon^{2} \sum_{r=1}^{1 / \varepsilon} r^{2} \phi(r) \approx \frac{3 n}{\pi^{2} \varepsilon^{2}} .
$$

Comparing the last two expressions, we obtain

$$
I \approx c n^{2 / 3} m^{2 / 3} \text { with } \quad c=\sqrt[3]{\frac{3}{4 \pi^{2}}} \approx 0.42 .
$$


We can also generalize Theorem 2 for multigraphs with bounded edge-multiplicity, improving the constant in Székely's result [S95].

Theorem 4.3. Let $G$ be a multigraph with maximum edge-multiplicity $m$. Then

$$
\operatorname{cr}(G) \geq \frac{1}{33.75} \frac{e^{3}(G)}{m v^{2}(G)}-0.9 m^{2} v(G) .
$$

Proof. Define a random simple subgraph $G^{\prime}$ of $G$ on the same vertex set. For each pair of vertices $v_{1}, v_{2}$ of $G$, let $e_{1}, e_{2}, \ldots e_{k}$ be the edges connecting them. With probability $1-k / m, G^{\prime}$ will not contain any edge between $v_{1}$ and $v_{2}$. With probability $k / m, G^{\prime}$ contains precisely one such edge, and the probability that this edge is $e_{i}$ is $1 / m(1 \leq i \leq k)$.

Applying Theorem 2 to $G^{\prime}$, the result follows.

\section{References}

[AC82] M. Ajtai, V. Chvátal, M. Newborn, E. Szemerédi, Crossing-free subgraphs, Ann. Discrete Mathematics 12 (1982), 9-12.

[CE90] K. Clarkson, H. Edelsbrunner, L. Guibas, M. Sharir, E. Welzl, Combinatorial complexity bounds for arrangements of curves and surfaces, Discrete and Computational Geometry 5 (1990), 99-160.

[HW54] G. H. Hardy, E. M. Wright, An Introduction to the Theory of Numbers, University Press, Oxford, 1954.

[L83] T. Leighton, Complexity Issues in VLSI, Foundations of Computing Series, MIT Press, Cambridge, MA, 1983.

[PA95] J. Pach and P.K. Agarwal, Combinatorial Geometry, John Wiley, New York, 1995.

[PS96] J. Pach and M. Sharir, On the number of incidences between points and curves, Combinatorics, Probability, and Computing, submitted.

[S96] J. Spencer, personal communication.

[S95] L. Székely, Crossing numbers and hard Erdös problems in discrete geometry, Combinatorics, Probability, and Computing, to appear.

[ST83] E. Szemerédi and W. T. Trotter, Extremal problems in discrete geometry, Combinatorica 3 (1983), 381-392. 